\title{
Correction to: Management of hyperglycaemia in type 2 diabetes, 2018. A consensus report by the American Diabetes Association (ADA) and the European Association for the Study of Diabetes (EASD)
}

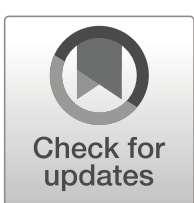

\author{
Melanie J. Davies ${ }^{1,2}$ • David A. D'Alessio ${ }^{3}$ - Judith Fradkin ${ }^{4}$ - Walter N. Kernan ${ }^{5}$. Chantal Mathieu ${ }^{6}$. \\ Geltrude Mingrone ${ }^{7,8,9} \cdot$ Peter Rossing ${ }^{10,11} \cdot$ Apostolos Tsapas $^{12} \cdot$ Deborah J. Wexler $^{13,14} \cdot$ John B. Buse ${ }^{15}$
}

Published online: 21 March 2019

(C) Springer-Verlag GmbH Germany, part of Springer Nature 2019

\section{Correction to: Diabetologia \\ https://doi.org/10.1007/s00125-018-4729-5}

The affiliation details for Geltrude Mingrone are corrected below.

The online version of the original article can be found at https://doi.org/ $10.1007 / \mathrm{s} 00125-018-4729-5$

\section{Melanie J. Davies}

Melanie.davies@uhl-tr.nhs.uk

1 Diabetes Research Centre, University of Leicester, Leicester, UK

2 Leicester Diabetes Centre, Leicester General Hospital, Leicester LE5 4PW, UK

3 Department of Medicine, Duke University School of Medicine, Durham, NC, USA

4 National Institute of Diabetes and Digestive and Kidney Diseases, National Institutes of Health, Bethesda, MD, USA

5 Department of Medicine, Yale School of Medicine, New Haven, CT, USA

6 Clinical and Experimental Endocrinology, UZ Gasthuisberg, KU Leuven, Leuven, Belgium
Fondazione Policlinico Universitario A. Gemelli IRCCS, Roma, Italia

8 Università Cattolica del Sacro Cuore, Roma, Italia

9 Diabetes and Nutritional Sciences, King's College London, London, UK

10 Steno Diabetes Center Copenhagen, Gentofte, Denmark

11 University of Copenhagen, Copenhagen, Denmark

12 Second Medical Department, Aristotle University Thessaloniki, Thessaloniki, Greece

13 Department of Medicine and Diabetes Unit, Massachusetts General Hospital, Boston, MA, USA

14 Harvard Medical School, Boston, MA, USA

15 Department of Medicine, University of North Carolina School of Medicine, Chapel Hill, NC, USA 